\title{
Quantitative ultrasound measurements of bone strength in female adolescent idiopathic scoliosis patients
}

\author{
H Wang ${ }^{1 *}, \mathrm{Q} \mathrm{Du}{ }^{1}$, PJ Chen ${ }^{2}, \mathrm{JA} \mathrm{Li}{ }^{3}, \mathrm{XH} \mathrm{He}{ }^{4}$ \\ From 9th International Conference on Conservative Management of Spinal Deformities - SOSORT 2012 \\ Annual Meeting \\ Milan, Italy. 10-12 May 2012
}

\section{Background}

The etiology of adolescent idiopathic scoliosis (AIS) has been linked to many factors, such as asymmetric growth, neuromuscular conditions and genetics. Several studies have been focused on the relationship between bone and scoliosis $[1,2]$. However, very few studies have examined the effect of AIS on bone mineralization.

\section{Aim}

The purpose of this study was to determine bone strength by quantitative ultrasound (QUS) measurements of bone speed of sound (SOS) along the longitudinal axis of the radial bones in scoliosis children.

\section{Methods}

This study was approved by the Human Research Ethic Committee of the School of Medicine, Jiaotong University, China. Eighty nine untreated female AIS patients were recruited in this study. The age range was from 10 to 16 years. Those who were using calcium supplements and those who had history of bone diseases were excluded from the study. The diagnoses of all AIS were confirmed by radiography based on American College of Radiology Guidelines and Recommendations. Cobb's angles ranged from $10-52^{\circ}$ (average $27.13^{\circ}$ ). The types of scoliosis included thoracic, lumbar, thoracolumbar and double curvatures. Patients' menstrual conditions and family history were also taken in consideration. SOS was measured (by Sunlight omnisense) in the distal 1/3 of the radial bones. Data were compared to age and gender-matched norms (standard SOS value provided by Sunlight system).

\section{Results}

Radial SOS was significantly reduced in AIS patients compared to non-AIS subjects $(\mathrm{P}<0.01)$; the radial SOS was also correlated to the age of the onset of menstrual cycle (lower in pre-menstrual cycle patients, $3626.92 \pm 124.35$ vs. $3702.68 \pm 192.23, \mathrm{P}<0.05)$. No significant correlations were found among the type of curvatures, the degree of Cobb's angle, family history and bone SOS $(\mathrm{P}<0.05)$.

\section{Conclusion}

Bone strength measured by QUS is reduced in AIS patients. The onset of menstrual cycle may have an effect on the reduction of SOS. Bone SOS is not affected by the types and severity of the scoliosis.

\section{Author details}

${ }^{1}$ Xinhua Hospital, Shanghai, China. ${ }^{2}$ Shanghai University of Sports, Shanghai, China. ${ }^{3}$ Jiangsu Province People's hospital, Jiangshu, China. ${ }^{4}$ Palmer College of Chiropractic, Florida, USA.

Published: 3 June 2013

\section{References}

1. Healey JH, Lane JM: Structural scoliosis in osteoporotic women. Clin Orthop Relat Res 1985, , 195: 216-223.

2. Thevenon A, Pollez B, Cantegrit F, Tison-Muchery F, Marchandise X, Duquesnoy B: Relationship between kyphosis, scoliosis, and osteoporosis in the elderly population. Spine (Phila Pa 1976) 1987, 12(8):744-745.

\section{doi:10.1186/1748-7161-8-S1-O7}

Cite this article as: Wang et al:: Quantitative ultrasound measurements of bone strength in female adolescent idiopathic scoliosis patients. Scoliosis 2013 8(Suppl 1):O7.

${ }^{1}$ Xinhua Hospital, Shanghai, China

Full list of author information is available at the end of the article

(c) 2013 Wang et al; licensee BioMed Central Ltd. This is an Open Access article distributed under the terms of the Creative Commons Attribution License (http://creativecommons.org/licenses/by/2.0), which permits unrestricted use, distribution, and reproduction in any medium, provided the original work is properly cited. 